\title{
Capillaria Ova and Diagnosis of Trichuris trichiura Infection in Humans by Kato-Katz Smear, Liberia
}

\section{Kerstin Fischer, Abakar Gankpala, Lincoln Gankpala, Fatorma K. Bolay, Kurt C. Curtis, Gary J. Weil, Peter U. Fischer}

We examined human stool samples from Liberia for soiltransmitted helminth ova by Kato-Katz smear and by quantitative PCR. Twenty-five samples were positive for Trichuris trichiura by smear but negative by quantitative PCR. Reexamination of samples showed that they contained Capillaria eggs that resemble T. trichiura in KatoKatz smears.

$\mathrm{K}$ ato-Katz smears are the most commonly used diagnostic tool for detecting and quantifying soil-transmitted helminth (STH) infections in field surveys (1). Although this method has some shortcomings, its advantages are field suitability and fast microscopic enumeration of worm eggs. Whereas sensitivity is low for light infections because of the small amount of stool examined $(\approx 41 \mathrm{mg}$ ), the specificity of Kato-Katz for diagnosis of Ascaris lumbricoides and Trichuris trichiura infection is considered to be high (2). In contrast, hookworm eggs are difficult to differentiate by morphology, but quantitative PCR (qPCR) enables differentiation among Necator americanus, Ancylostoma duodenale, and A. ceylanicum eggs (3-5).

Among helminth eggs found in human feces, the barrel-shaped eggs of T. trichiura worms are considered to be characteristic, with a length of $50-55 \mu \mathrm{m}$, a width of 22-24 $\mu \mathrm{m}$, and clearly protruding bipolar plugs (6). Similar eggs of other members of the Trichiuridae family may be differentiated from $T$. trichiura eggs by size and morphology when observed at high magnification, but these eggs have rarely been found in human fecal samples (7-9). Therefore, the presence of eggs of zoonotic members of the Trichiuridae family is generally not considered a confounder for detecting $T$. trichiura by Kato-Katz smear.

Author affiliations: Washington University School of Medicine,

St. Louis, Missouri, USA (K. Fischer, K.C. Curtis, G.J. Weil,

P.U. Fischer); National Public Health Institute of Liberia,

Charlesville, Liberia (A. Gankpala, L. Gankpala, F.K. Bolay)

DOI: https://doi.org/10.3201/eid2408.180184

\section{The Study}

To assess the effect of mass drug administration using ivermectin and albendazole for the elimination of lymphatic filariasis on STH prevalence and intensity, we collected stool samples over a period of 3 years in 2 different areas in Foya district (Lofa County) in northwestern Liberia and in Harper district (Maryland County) in southeastern Liberia (10). We examined a single stool sample per subject by microscopy (magnification $\times 100$ ) with duplicate Kato-Katz smears (41-mg template). We preserved aliquots of randomly selected specimens on FTA cards (GE Healthcare, Little Chalfont, UK) or in RNAlater (ThermoFisher, Waltham, MA, USA) and shipped them to Washington University School of Medicine (St. Louis, MO, USA) for analysis by qPCR. Two experienced microscopists (L.G., A.T. Momolu) examined the samples by Kato-Katz smear in both study areas. For detection of STH by qPCR, we extracted DNA from $\approx 100 \mathrm{mg}$ of stool and tested it as described by Pilotte et al. (5) with a Quantstudio 6 Flex Thermocycler (Applied Biosystems, Carlsbad, CA, USA) and TaqMan Fast Advanced Mastermix (Applied Biosystems). We used the following primers and probes to detect Schistosoma mansoni DNA: forward primer 5'-TGTGGGAGTCTTTGGTTGTT-3', reverse primer 5'-CAACATGACTGGGAACAGGA-3', probe 5'-AGGTTCAGGTGG/ZEN/GTGTGTTACGAA-31ABkFQ-3'.

We tested 353 stool samples from Foya district by Kato-Katz smear; 31 (8.8\%) were positive for $A$. lumbricoides eggs, 231 (65.4\%) for hookworm eggs, 27 (7.6\%) for $T$. trichiura-like eggs, and 276 (78.2\%) for S. mansoni eggs. We tested 225 samples from Harper district by Kato-Katz smear; 163 (72.4\%) were positive for A. lumbricoides eggs, 65 (28.9\%) for hookworm eggs, and 51 (22.7\%) for T. trichiura eggs (Table 1). There was good agreement between the results of the Kato-Katz and qPCR tests for the specimens from Harper (80.5\%-91.6\%), but generally qPCR had higher sensitivity. Our results were consistent with results previously reported with samples from other areas $(3,11)$. Agreement between the 2 diagnostic tests for samples from Foya ranged from $77.3 \%$ to $92.9 \%$, but the sensitivity of the qPCR was unexpectedly low, a finding that was especially true for Ascaris and Trichuris infection (Table 1). Whereas samples positive for Ascaris by Kato-Katz but negative by qPCR had low egg counts, samples positive for Trichuris by Kato-Katz 
Table 1. Comparison of sensitivity of Kato-Katz smear and quantitative PCR results for 778 stool samples tested for soil-transmitted helminths, Foya and Harper districts, Liberia

\begin{tabular}{lcccc}
\hline Site and species & No. positive* & Kato-Katz smear sensitivity, \% & qPCR sensitivity, \% & McNemar $\mathrm{p}$ value \\
\hline Foya district, $\mathrm{n}=353$ & & & & \\
Ascaris lumbricoides & 34 & 91.2 & 17.6 & $<0.0001$ \\
Hookworm† & 247 & 93.5 & 7.4 & $<0.0001$ \\
Trichuris trichiura & 27 & 100 & 84.0 & $<0.0001$ \\
Schistosoma mansoni & 307 & 89.9 & & 0.0573 \\
\hline Harper district, $\mathrm{n}=225$ & 180 & & 98.9 & 0.0013 \\
A. lumbricoides & 99 & 90.6 & 89.9 & 0.0005 \\
Hookworm† & 86 & 59.7 & 94.2 & 0.0001 \\
T. trichiura & & & & 8.3 \\
\hline
\end{tabular}

*Samples that tested positive by either method.

†Hookworm was Necator americanus. No Ancylostoma duodenale was detected.

but negative by qPCR had higher counts; 7 samples contained $\geq 1,000$ barrel-shaped eggs/g of stool (Table 2). We repeated DNA extraction and qPCR and also used an alternative qPCR for T. trichiura (3), but these tests did not improve the agreement between microscopy and qPCR results.

To check further whether Kato-Katz-positive, qPCRnegative stool samples contained T. trichiura eggs, we examined direct smears of stool samples preserved in RNAlater by microscopy (magnification $\times 100$ and $\times 400$ ) (Figure 1). The samples positive by qPCR contained eggs ( 6 measured) with typical T. trichiura morphology; these eggs had a mean $( \pm \mathrm{SD})$ length of $52 \mu \mathrm{m}( \pm 2.4 \mu \mathrm{m})$ and width of $25.5 \mu \mathrm{m}( \pm 1.3$ $\mu \mathrm{m})$. In contrast, qPCR-negative samples contained eggs (31 measured) with a mean $( \pm \mathrm{SD})$ length of $51.8 \mu \mathrm{m}( \pm 1.5 \mu \mathrm{m})$ and width of $32.7 \mu \mathrm{m}( \pm 2.1 \mu \mathrm{m})$. The qPCR-negative samples also had less pronounced plugs and a thick, striated shell, features that are consistent with eggs of Capillaria hepatica (syn. Calodium hepaticum) and some other Capillaria species (Trichuridae). Eggs of C. philippinensis or C. aerophila that have been observed in human stool samples previously were either smaller or larger than the Capillaria eggs found in Lofa $(12,13)$. Because polar plugs of these eggs are less prominent than those of T. trichiura, and because their shapes are sometimes more oval or round, they can also be confused with $A$. lumbricoides eggs by low-power microscopy, especially if only a few eggs were detected (Figure 1).

Members of the subfamily Capillaridae are animal parasites with somewhat divergent life cycles, and most

\begin{tabular}{|c|c|c|c|c|c|c|c|c|c|c|c|}
\hline \multicolumn{4}{|c|}{ Demographics } & \multicolumn{4}{|c|}{ Microscopy, epg } & \multicolumn{4}{|c|}{ qPCR, cycle threshold } \\
\hline Year & Patient no. & Age, y/sex & Village & $\mathrm{Tt}$ & $\mathrm{Al}$ & $\mathrm{Hk}$ & $\mathrm{Sm}$ & $\mathrm{Tt}$ & $\mathrm{Al}$ & $\mathrm{Na}$ & Sm \\
\hline \multirow[t]{8}{*}{2014} & P320529 & $45 / F$ & Yallahun & 576 & 0 & 360 & 24 & Neg & Neg & 31.7 & 30.5 \\
\hline & P320683 & $35 / F$ & Kpombu & 12 & 0 & 0 & 0 & $\mathrm{Neg}$ & $\mathrm{Neg}$ & $\mathrm{Neg}$ & 28.4 \\
\hline & P320695 & 16/M & Kpombu & 24 & 0 & 0 & 72 & Neg & Neg & Neg & 23.8 \\
\hline & P320620 & 15/M & Foya-Dundu & 12 & 120 & 0 & 288 & Neg & Neg & 32.2 & 23.5 \\
\hline & P320746 & 9/F & Bandenin & 24 & 0 & 0 & 0 & $\mathrm{Neg}$ & Neg & Neg & 26.51 \\
\hline & P320452 & $7 / F$ & Felaloe & 12 & 0 & 0 & 120 & Neg & Neg & Neg & 23.9 \\
\hline & P320596 & $6 / F$ & Foya-Dundu & 12 & 0 & 0 & 90 & Neg & Neg & Neg & 27.6 \\
\hline & P320656 & $6 / \mathrm{F}$ & Kpombu & 120 & 0 & 0 & 504 & $\mathrm{Neg}$ & Neg & $\mathrm{Neg}$ & 21.3 \\
\hline \multirow[t]{7}{*}{2016} & P331772 & $36 / \mathrm{M}$ & Kpormbu & 3,048 & 0 & 12 & 24 & Neg & Neg & Neg & Neg \\
\hline & P331921 & 35/M & Felaloe & 60 & 0 & 0 & 12 & Neg & Neg & Neg & Neg \\
\hline & P331783 & $34 / F$ & Kpormbu & 420 & 0 & 0 & 0 & $\mathrm{Neg}$ & $\mathrm{Neg}$ & $\mathrm{Neg}$ & Neg \\
\hline & P330724 & $26 / \mathrm{M}$ & Keyabendu & 4,224 & 0 & 0 & 456 & $\mathrm{Neg}$ & $\mathrm{Neg}$ & $\mathrm{Neg}$ & 30.4 \\
\hline & P331791 & $6 / F$ & Kpormbu & 12 & 0 & 156 & 12 & $\mathrm{Neg}$ & $\mathrm{Neg}$ & 33.1 & 33.4 \\
\hline & P331962 & $6 / F$ & Bandenin & 12 & 0 & 0 & 168 & $\mathrm{Neg}$ & $\mathrm{Neg}$ & $\mathrm{Neg}$ & 29.6 \\
\hline & P331983 & $6 / F$ & Bandenin & 36 & 0 & 0 & 5,304 & $\mathrm{Neg}$ & $\mathrm{Neg}$ & $\mathrm{Neg}$ & 28.1 \\
\hline \multirow[t]{12}{*}{2017} & P341287 & $61 / \mathrm{M}$ & Mendikorma & 1,464 & 0 & 0 & 0 & Neg & Neg & Neg & 33.1 \\
\hline & P341282 & $56 / \mathrm{M}$ & Mendikorma & 540 & 0 & 216 & 0 & Neg & $\mathrm{Neg}$ & 28.3 & $\mathrm{Neg}$ \\
\hline & P341284 & $50 / \mathrm{M}$ & Mendikorma & 60 & 0 & 0 & 132 & Neg & $\mathrm{Neg}$ & Neg & $\mathrm{Neg}$ \\
\hline & P342148 & $45 / \mathrm{M}$ & Keyabendu & 1,368 & 0 & 0 & 192 & Neg & Neg & 34.5 & Neg \\
\hline & P340246 & $39 / \mathrm{M}$ & Kamatahun & 120 & 0 & 0 & 216 & $\mathrm{Neg}$ & $\mathrm{Neg}$ & $\mathrm{Neg}$ & 30.0 \\
\hline & P340307 & $19 / F$ & Bambuloe & 2,028 & 0 & 0 & 1,188 & Neg & $\mathrm{Neg}$ & Neg & 24.1 \\
\hline & P340133 & $12 / \mathrm{M}$ & Fokolahun & 1,020 & 16,392 & 0 & 0 & 25.3 & 16.7 & $\mathrm{Neg}$ & $\mathrm{Neg}$ \\
\hline & P340183 & 9/F & $\begin{array}{l}\text { Kpelloe } \\
\text { Ndama }\end{array}$ & 72 & 0 & 0 & 0 & Neg & Neg & Neg & 36.0 \\
\hline & P341308 & 9/F & Mendikorma & 36 & 0 & 108 & 0 & $\mathrm{Neg}$ & Neg & $\mathrm{Neg}$ & 28.5 \\
\hline & P341326 & $9 / M$ & Mendikorma & 456 & 0 & 0 & 0 & Neg & Neg & 26.5 & 30.4 \\
\hline & P341327 & $6 / M$ & Mendikorma & 2,076 & 0 & 0 & 0 & $\mathrm{Neg}$ & $\mathrm{Neg}$ & Neg & Neg \\
\hline & P340147 & $5 / M$ & Fokolahun & 48 & 0 & 0 & 0 & 30.94 & 26.93 & Neg & Neg \\
\hline
\end{tabular}



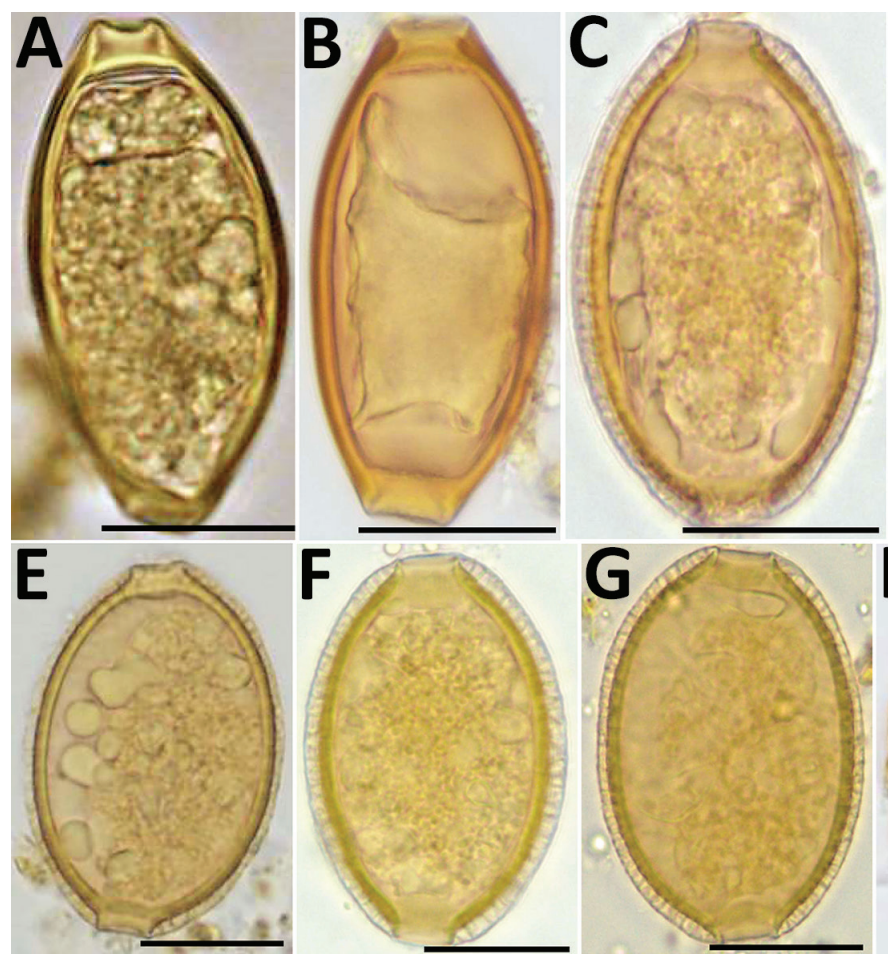

do not infect humans. Pseudoinfections with $C$. hepatica occur; eggs found in stool are present because they were consumed in infected animal liver. However, actual infections with $C$. hepatica do not lead to the passing of eggs in stool (9). Other species such as $C$. philippinensis cause true infections (and autoinfection) with eggs found in stool; the infection is linked to consumption of raw fish. Human capillariasis has not been reported from Liberia, and only isolated case reports have been published from sub-Saharan Africa (7-9). We performed DNA sequencing to better characterize the Capillaria species found in Foya. Using the primers Kt875351.1 (5'-CCCTAGTTGCGACTTTAAACGA-3') and Capillaria 18S1R (5'- TCCACCAACTAAGAACGGCC-3'), we were able to amplify and sequence a 288-bp portion of the $18 \mathrm{~S}$ rDNA from $T$. trichiura qPCR-negative samples that contained only eggs morphologically identified as Capillaria spp. (GenBank accession no. MG859285). The DNA fragment was $100 \%$ identical to orthologs of $C$. hepatica (accession no. MF287972.1), Aonchotheca putorii (C. putorii) (accession no. LC052356.2), and Pearsonema plica (C. plica) (accession no. MF621034.1), Capillaria worm species that have varying life cycles and host species but that are only $95 \%$ identical to the ortholog of $T$. trichiura.

The life cycle and the medical importance of the Capillaria species found in humans in northwestern Liberia remain to be elucidated. In our study some subjects showed high Capillaria egg loads that may indicate a true infection rather than pseudoinfection. However, transient high egg

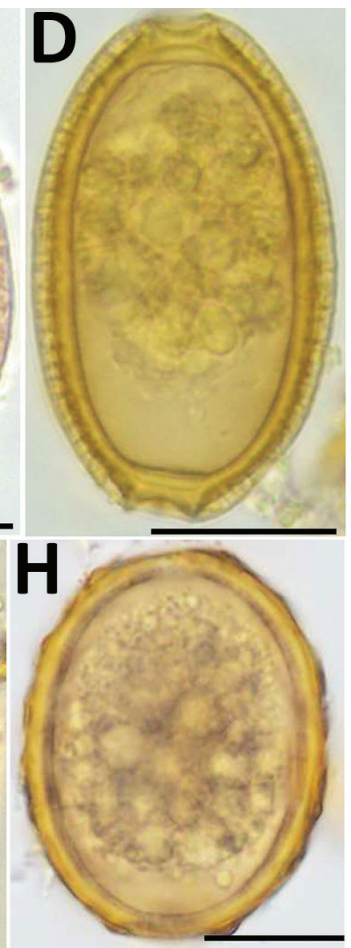

Figure. Helminth eggs found in stool samples from persons in Lofa County, Liberia. A, B) Eggs of Trichuris trichiura in samples positive for T. trichiura by Kato-Katz smear and by qPCR. C-F) Eggs of Capillaria spp. in samples positive for T. trichiura by Kato-Katz smear but negative for T. trichiura by qPCR. G) Egg of Capillaria spp. in sample positive for Ascari lumbricoides by Kato-Katz smear but negative for $A$. lumbricoides by qPCR. $\mathrm{H}$ ) Egg of $A$. lumbricoides in sample positive for $A$. lumbricoides by Kato-Katz smear and qPCR. Scale bars indicate $20 \mu \mathrm{m}$. qPCR, quantitative PCR.

counts have been reported in persons with pseudoinfections (7). Whereas consumption of bush meat in Foya is common, consumption of raw or undercooked fish, which is necessary for transmission of C. philippinesis, is rare.

\section{Conclusions}

This study shows that Capillaria eggs similar to those of C. hepatica are not uncommon in stool samples collected in Liberia. These eggs can be misidentified by Kato-Katz smear as T. trichiura or as A. lumbricoides, which can confound results of STH surveys. The misidentification can also lead to an incorrect assumption that antihelminthic treatment was ineffective. Our results also illustrate the value of qPCR for validating Kato-Katz test results and for explaining unexpected findings.

\section{Acknowledgments}

We thank Emanuel B. Gray and Aaron T. Momolu for their expert help during field work, as well as Laura Rinaldi and Guiseppe Cringoli for their advice concerning animal Capillaria species.

The study was funded in part by grant GH5341 from the Bill \& Melinda Gates Foundation.

\section{About the Author}

Ms. Fischer is a medical technician and staff scientist at the Division of Infectious Diseases, Department of Medicine, Washington University School of Medicine, St. Louis, Missouri, USA. For the last 20 years, her research has focused on medical helminthology and tropical medicine. 


\section{References}

1. World Health Organization. Prevention and control of schistosomiasis and soil-transmitted helminthiasis. WHO Technical Report Series No. 912. Geneva: The Organization; 2002.

2. Speich B, Ali SM, Ame SM, Albonico M, Utzinger J, Keiser J. Quality control in the diagnosis of Trichuris trichiura and Ascaris lumbricoides using the Kato-Katz technique: experience from three randomised controlled trials. Parasit Vectors. 2015;8:82. http://dx.doi.org/10.1186/s13071-015-0702-z

3. Mejia R, Vicuña Y, Broncano N, Sandoval C, Vaca M, Chico M, et al. A novel, multi-parallel, real-time polymerase chain reaction approach for eight gastrointestinal parasites provides improved diagnostic capabilities to resource-limited at-risk populations. Am J Trop Med Hyg. 2013;88:1041-7. http://dx.doi.org/10.4269/ ajtmh.12-0726

4. Papaiakovou M, Pilotte N, Grant JR, Traub RJ, Llewellyn S, McCarthy JS, et al. A novel, species-specific, real-time PCR assay for the detection of the emerging zoonotic parasite Ancylostoma ceylanicum in human stool. PLoS Negl Trop Dis. 2017;11:e0005734. http://dx.doi.org/10.1371/journal.pntd.0005734

5. Pilotte N, Papaiakovou M, Grant JR, Bierwert LA, Llewellyn S, McCarthy JS, et al. Improved PCR-based detection of soil transmitted helminth infections using a next-generation sequencing approach to assay design. PLoS Neg1 Trop Dis. 2016;10:e0004578. http://dx.doi.org/10.1371/journal.pntd.0004578

6. World Health Organization. Training manual on the diagnosis of intestinal parasites based on the WHO bench aids for diagnosis of intestinal parasites. Geneva: The Organization; 2004. p. 1-48.

7. Cabada MM, Lopez M, White AC Jr. Capillaria hepatica pseudoinfection. Am J Trop Med Hyg. 2013;89:609. http://dx.doi.org/10.4269/ajtmh.13-0126

8. El-Dib NA, El-Badry AA, Ta-Tang TH, Rubio JM. Molecular detection of Capillaria philippinensis: An emerging zoonosis in Egypt. Exp Parasitol. 2015;154:127-33. http://dx.doi.org/ 10.1016/j.exppara.2015.04.011

9. Fuehrer HP, Igel P, Auer H. Capillaria hepatica in man-an overview of hepatic capillariosis and spurious infections. Parasitol Res. 2011;109:969-79. http://dx.doi.org/10.1007/ s00436-011-2494-1

10. Chesnais CB, Awaca-Uvon NP, Bolay FK, Boussinesq M, Fischer PU, Gankpala L, et al. A multi-center field study of two point-of-care tests for circulating Wuchereria bancrofti antigenemia in Africa. PLoS Negl Trop Dis. 2017;11:e0005703. http://dx.doi.org/10.1371/journal.pntd.0005703

11. Easton AV, Oliveira RG, O’Connell EM, Kepha S, Mwandawiro CS, Njenga SM, et al. Multi-parallel qPCR provides increased sensitivity and diagnostic breadth for gastrointestinal parasites of humans: field-based inferences on the impact of mass deworming. Parasit Vectors. 2016;9:38. http://dx.doi.org/10.1186/s13071-016-1314-y

12. Attia RAH, Tolba MEM, Yones DA, Bakir HY, Eldeek HE, Kamel S. Capillaria philippinensis in Upper Egypt: has it become endemic? Am J Trop Med Hyg. 2012;86:126-33. http://dx.doi.org/10.4269/ajtmh.2012.11-0321

13. Di Cesare A, Castagna G, Meloni S, Otranto D, Traversa D. Mixed trichuroid infestation in a dog from Italy. Parasit Vectors. 2012;5:128. http://dx.doi.org/10.1186/ $1756-3305-5-128$

Address for correspondence: Peter U. Fischer, Washington University School of Medicine-Infectious Diseases Division, Department of Medicine, 4444 Forest Park Blvd, St. Louis, MO 63108, USA; email: Pufischer@wustl.edu

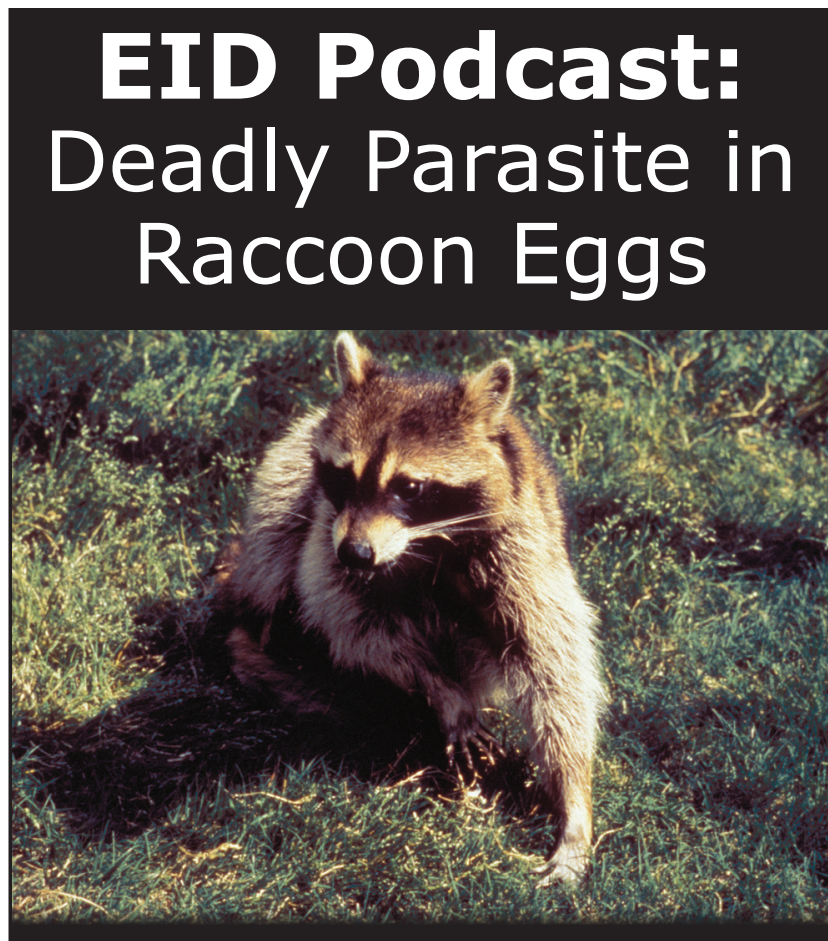

Infection with Baylisascaris procyonis roundworms is rare but often fatal and typically affects children.

Baylisascaris procyonis, the common intestinal roundworm of raccoons, has increasingly been recognized as a source of severe, often fatal, neurologic disease in humans, particularly children. Although this devastating disease is rare, lack of effective treatment and the widespread distribution of raccoons in close association with humans make baylisascariasis a disease that seriously affects public health. Raccoons infected with $B$. procyonis roundworms can shed millions of eggs in their feces daily. Given the habit of raccoons to defecate in and around houses, information about optimal methods to inactivate $B$. procyonis eggs are critical for the control of this disease. However, little information is available about survival of eggs and effective disinfection techniques. Additional data provide infomation on thermal death point and determining the impact of desiccation and freezing on the viability of $B$. procyonis eggs to provide additional information for risk assessments of contamination and guide attempts at environmental decontamination.

Visit our website to listen: https: / / www2c.cdc.gov/ podcasts/ player.asp $\mathbf{f}=\mathbf{8 6 2 0 6 7 5}$

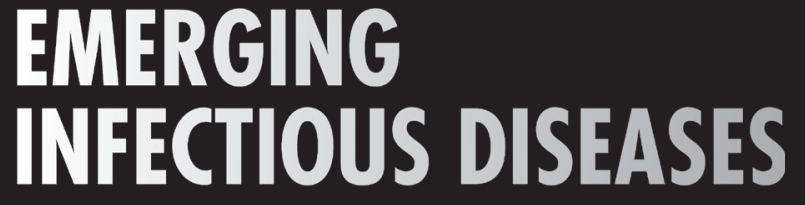

